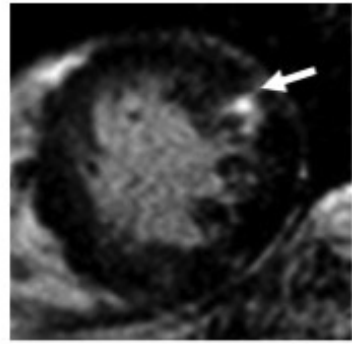

Baseline

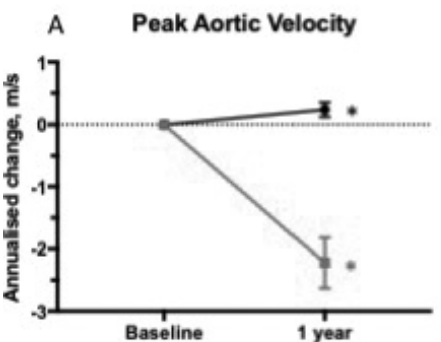

D Extracellular Volume Fraction

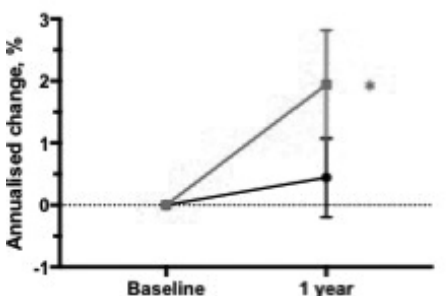

* $\mathrm{P}<0.05$ (baseline compared to annualised 1 year)

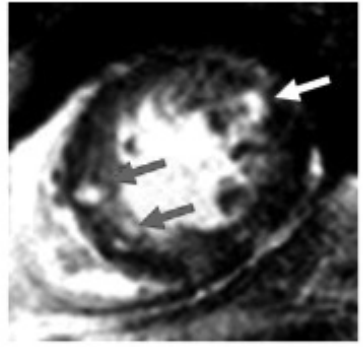

1 Year

B Indexed Left Ventricular Mass

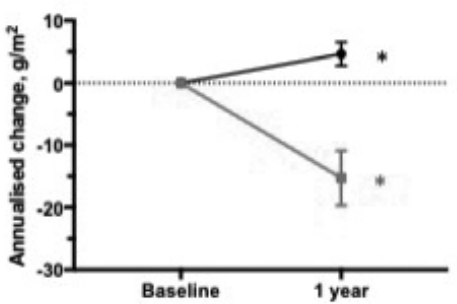

E Indexed Extracellular Volume

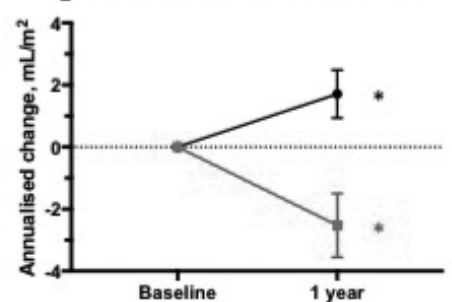

- Natural History

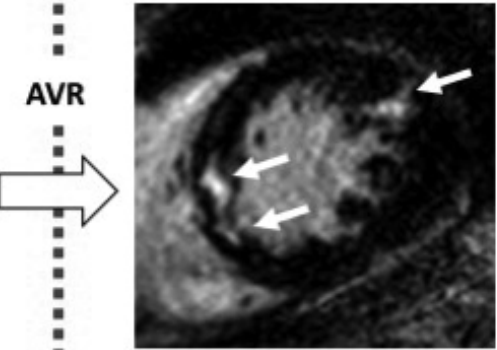

2 Year
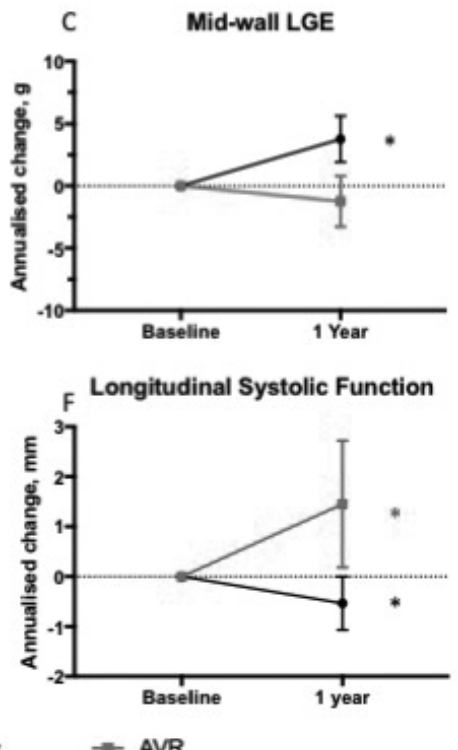

Abstract 012 Figure 1 Main Figure: Serial cardiac magnetic resonance imaging in a patient with severe aortic stenosis.

hypertrophy with more gradual reduction in diffuse fibrosis burden but no change in replacement fibrosis.

Mid-wall LGE is present on baseline scan (white arrow) and new areas are seen on 1 year scan (red arrows). Following AVR there is no change in mid-wall LGE (white arrows). Annualised change in imaging measures is presented below in the natural history (black) and AVR (red) groups.

\section{FREE-BREATHING MOCO LGE LEADS TO BETTER IMAGE QUALITY AND FASTER SCANNING TIMES IN CLINICAL PRACTICE}

${ }^{1}$ Ilaria Lobascio, ${ }^{1,2}$ Gabriella Captur, ${ }^{1}$ Veronica Culotta, ${ }^{3}$ Redha Boubertakh, ${ }^{4}$ Andras Eke, ${ }^{5}$ Hui Xue, ${ }^{1,6}$ Charlotte Manisty, ${ }^{5}$ Peter Kellman, ${ }^{1,6}$ James C Moon. ${ }^{1}$ Barts Heart Centre, The Cardiovascular Magnetic Resonance Imaging Unit, St Bartholomew's Hospital, West Smithfield, London, UK; ${ }^{2}$ UCL Biological Mass Spectrometry Laboratory, Institute of Child Health and Great Ormond Street Hospital, London and NIHR University College London Hospitals Biomedical Research Centre, London W1T, UK; ${ }^{3}$ Cardiovascular Biomedical Research Unit, Barts and the London School of Medicine and Dentistry, Queen Mary University of London, UK; ${ }^{4}$ Department of Physiology, Semmelweis University, Budapest, Hungary and Diagnostic Radiology, Yale University, New Haven, CT, USA; ${ }^{5}$ National Heart, Lung, and Blood Institute, National Institutes of Health, DHHS, Bethesda, MD, USA; ${ }^{6}$ University College London UCL Institute of Cardiovascular Science, London WC1E 6BT, UK

10.1136/heartjnl-2017-311399.13

Objectives Late gadolinium enhancement (LGE) sequences have evolved. Free-breathing, motion-corrected LGE (MOCO-
LGE) ${ }^{1,2}$ has several potential advantages over breath held LGE (bh-LGE) $^{3}$ including minimal user input for the LGE short axis (SA) stack and no need for breath-holds. We hypothesised that the use of MOCO-LGE would be faster, cheaper and easier for clinical scanning, increasing throughput.

Methods 200 consecutive clinical patients underwent bh-LGE or MOCO-LGE at $1.5 \mathrm{~T}$. Image quality (Figure 1), scan time, patient throughput (change-over time) and reader confidence were compared. LGE image quality was evaluated qualitatively (adaption of previously reported method) ${ }^{4}$ and quantitatively (assessing image texture heterogenity using grayscale lacunarity, $\lambda)$.

Results MOCO-LGE image quality was better than bh-LGE qualitatively (lower score better: $0.56 \pm 1.2$ vs $1.93 \pm 0.83$, $\mathrm{p}<0.0001)$ especially in clinically vulnerable patients eg. atrial fibrillation, poor breath-holding, low ejection fraction (0.59 vs $3.05, p=0.0001)$. Excellent image quality (score $=0$ ) was also more common $(78 \%$ vs $27 \%, \mathrm{p}<0.0001)$. Quantitative image quality was superior with MOCO-LGE (lower score better: blood pool lambda bh-LGE $0.38 \pm 0.11$ vs MOCO-LGE $0.28 \pm 0.08, \mathrm{p}<0.0001)$. MOCO-LGE led to greater diagnostic confidence (blinded review: basic analytic, retained diagnostic and "Omary" correction methods, respectively $\mathrm{p}=0.005$; $\mathrm{p}=0.018, \mathrm{p}<0.001)$. Although patient change-over time did not differ significantly between scan protocols, total LGE imaging time was 1.6 times shorter with MOCO-LGE compared to bh-LGE (5.23 vs 8.84 minutes, $\mathrm{p}<0.0001)$. 


\begin{tabular}{lccccc} 
Late Gadolinium Enhancement & 0 & 1 & 2 & 3 & Maximum score \\
\hline LV coverage & Full coverage & - & Apex not covered & Base or $\geq 1$ slice missing & 5 \\
Wrap around & No & 1 slice & 2 slices & $\geq 3$ slices & 3 \\
Respiratory Ghost & No & 1 slice & 2 slices & $\geq 3$ slices & 3 \\
Cardiac Ghost & No & 1 slice & 2 slices & $\geq 3$ slices & 3 \\
Image blurring/mis-trigger & No & 1 slice & 2 slices & $\geq 3$ slices & 3 \\
Metallic Artifacts & No & 1 slice & 2 slices & $\geq 3$ slices & 3 \\
Signal loss (coil inactive) & Activated & - & Not activated & - & 2 \\
Slice thickness & $\geq 10 \mathrm{~mm}$ & $11-15 \mathrm{~mm}$ & - & $>15 \mathrm{~mm}$ & 3 \\
Inter-slice Gap & $<3 \mathrm{~mm}$ & $3-4 \mathrm{~mm}$ & - & $>4 \mathrm{~mm}$ & 3 \\
Correct LV long axis & $\geq 3$ & 2 & 1 & None & 3 \\
LGE Score & & & & & 31 \\
\hline
\end{tabular}

Abstract 013 Figure 1 Adapted Quality Scoring Method for LGE images in CMR: 10 criteria; range of scores 0 (optimal quality) to 31 (poorest quality).

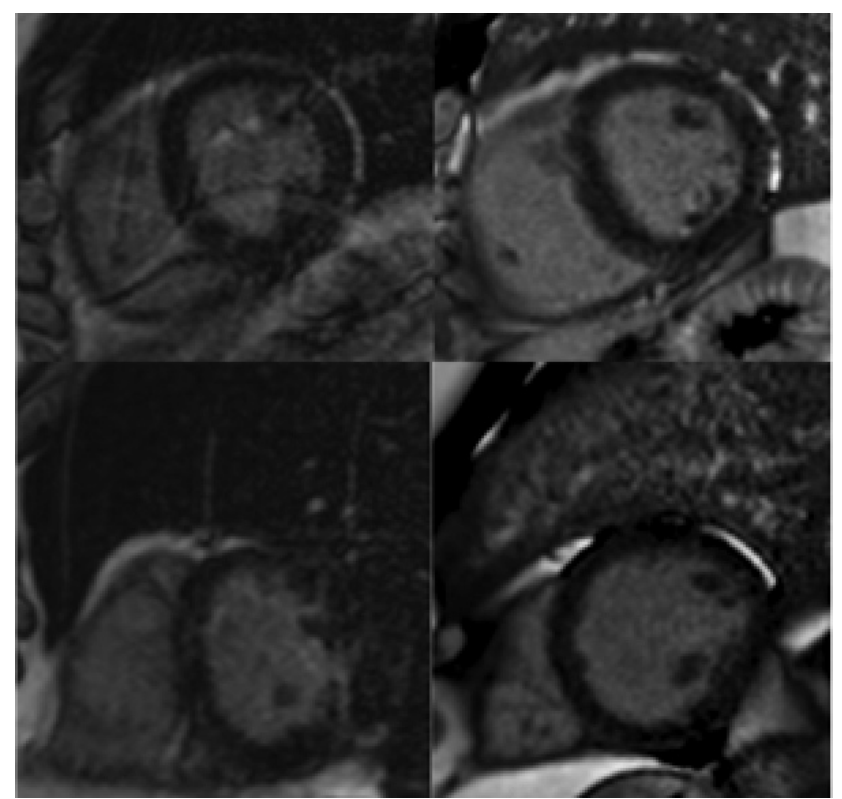

Abstract 013 Figure 2 Example images from a single patient showing bh-LGE image (left) with corresponding MOCO-LGE (right).

Conclusion MOCO-LGE is superior to bh-LGE in a clinical service, with better image quality, easier interpretation and faster scanning times.

\section{REFERENCES}

1. Maria JLedesma-Carbayo, Peter Kellman, Andrew EArai, et al. Motion corrected free-breathing delayed-enhancement imaging of myocardial infarction using nonrigid registration. JMRI Published Online First : 20 Jul 2007.

2. Peter Kellman, Andrew EArai. Cardiac imaging techniques for physicians: Late enhancement. JMRI Published Online First : 17 Aug 2012.

3. Piehler KM, Wong TC, Puntil KS, et al. Free-breathing, motion-corrected late gadolinium enhancement is robust and extends risk stratification to vulnerable patients. Circ Cardiovasc Imaging 2013;6(3):423-32.

4. Vincenzo Klinke, Stefano Muzzarelli, Nathalie Lauriers, et al. Quality assessment of cardiovascular magnetic resonance in the setting of the European CMR registry: description and validation of standardized criteria. I Cardiovasc Magn Reson 2013;15:55.

\section{WIDEBAND FREE BREATHING MOCO LGE CHANGES PATIENT CARE IN PATIENTS WITH IMPLANTABLE CARDIAC DEFIBRILLATORS}

Anish Bhuva, Manish Ramlall, Redha Boubertakh, Kristopher Knott, Patricia Feuchter, Neha Sekhri, Richard Schilling, Peter Kellman, James Moon, Charlotte Manisty. Barts Heart Centre, UK

\subsection{6/heartjnl-2017-311399.14}

Background There is a growing need for CMR in patients with implanted devices, who represent a high-risk cohort. Recent reports suggest CMR may be safely performed but artefact remains a significant limitation to late gadolinium enhancement (LGE). It is still unknown whether novel sequences to reduce artefact can be used efficiently or provide clinically useful information from scar imaging.

Methods We used a novel free- breathing wideband MOCO sequence with PSIR (WB-MOCO) designed for a clinical environment. Patients with implantable cardiac devices (including MR non-conditional) referred clinically for CMR were scanned according to local standard operating procedure (based on national guidelines and Magnasafe Registry). WBMOCO LGE approach was used primarily, with paired comparator conventional PSIR FLASH LGE or free breathing MOCO SSFP. Conventional and WB-MOCO LGE were assessed for artefact on a scale of 0 to $4 \quad(0=$ no artefact, $4=$ completely obscured). A panel of three CMR cardiologists judged impact on patient care.

Results Of the 67 patients (age 54 \pm 19 years, 47 male), 17 had ICDs, 9 cardiac resynchronisation devices, 19 pacemakers and 22 implantable loop recorders (ILR).

$20(44 \%)$ pulse generators were non-conditional; $11(16 \%)$ inpatients; 10 (15\%) with AF; 7 (10\%) pacing dependent. Every patient referred was scanned successfully. 10 leads had parameter changes by Magnasafe criteria with no clinical significance, and $80 \%$ normalising at follow up (66 \pm 40 days).

With conventional LGE imaging, 22 (33\%) scans were nondiagnostic. WB-MOCO LGE completely removed artefact in $19(87 \%)$, and achieved diagnosis in the remaining 3. 10 (15\%) patients had significant artefact on conventional LGE, which WB completely or almost completely removed. 